\title{
Two Case Reports of C-Shaped Mandibular Premolars with Three and Four Root Canals
}

\author{
Mohammed Sarhan Alzahrani*
}

Endodontics Department, Albaha University, Saudi Arabia

*Corresponding author: Mohammed Sarhan Alzahrani, Assistant Professor, Endodontic Consultant, Endodontics RDS Department, Albaha University, Albaha, Saudi Arabia, Tel: 00966543291242

\begin{abstract}
Commonly, the mandibular premolars' root canal system is complex and usually requires special management skills in root canal treatment. The occurrence of three root canals in the mandibular first premolar is relatively uncommon. Furthermore, the four root canals in the second premolar are extremely rare and clinically quite challenging to manage. Proper knowledge of the root canal anatomy and delicate radiographic interpretation and clinical inspection is essential for successful root canal treatment of the mandibular premolars. Skillful and special root canal preparation and obturating techniques are usually needed. Therefore, this paper reports two unusual cases of C-shaped mandibular premolars. The first case is a mandibular first premolar with three root canals. While the second case is a mandibular second premolar with four root canalsthat were successfully managed endodontically.
\end{abstract}

\section{Keywords}

Root canal treatment, Mandibular premolars, Root canal anatomy

\section{Introduction}

Although the mandibular first premolar is a single-rooted tooth $(97.21 \%)$, their canal configurations may vary from a single canal in (73.55\%) to two canals in (23.55\%). The reported incidence of three or more canals in the mandibular premolars is very uncommon $(0-5 \%)[1,2]$. C-shaped canals anatomy also has been reported in the mandibular premolars [3]. Several studies reported a frequency of $\sim 2.7 \%$ of two rooted mandibular first premolar teeth [4-6]. The occurrence of three-rooted mandibular first premolars is rare and occasionally reported in the case reports $[7,8]$.
Developmental grooves are usually present on both the mesial and distal surfaces of the mandibular first premolar root, resulting in an oval shaped root. The complexity of root canal anatomy is strongly associated with the presence and extent of root grooves [9]. Many studies have shown a strong association between the C-shaped canal and the deep radicular groove $[9,10]$. A Micro-CT study of the mandibular first premolar showed that $40.9 \%$ of the study sample's teeth had radicular grooves that were primarily located on the mesial side of the root (69.5\%) and were related to the teeth with complex root canal anatomy [11]. Ethnic variations in root canal anatomy were reported in a study that found a higher incidence of two root canals in African American than Caucasian groups of patients [12].

On the other hand, the mandibular second premolar is typically a single-rooted tooth (99.28\%). Two-rooted mandibular second premolar comprised $0.5 \%$ of the teeth. Three-rooted mandibular second premolar forms were rare and comprised $0.1 \%$ of teeth [13]. The developmental root groove more frequently presents on the distal root surface (73\%) [14]. Ethnic variation of the root canal anatomy was also reported in the mandibular second premolar. The incidence of two root canals in the mandibular second premolar was higher in African American patients than a Caucasian group of patients [12].

Moreover, mandibular second premolars usually have one root canal in (86.9\%) of the cases.More than three canals in mandibular second premolars were very rarely reported $(0-2 \%)[1,2]$. When a second canal system is present, it is more frequently toward the lingual

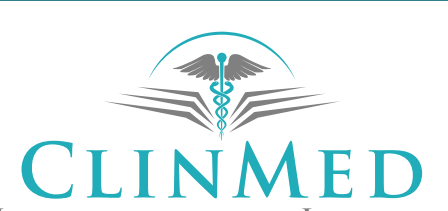

INTERNATIONAL LIBRARY 


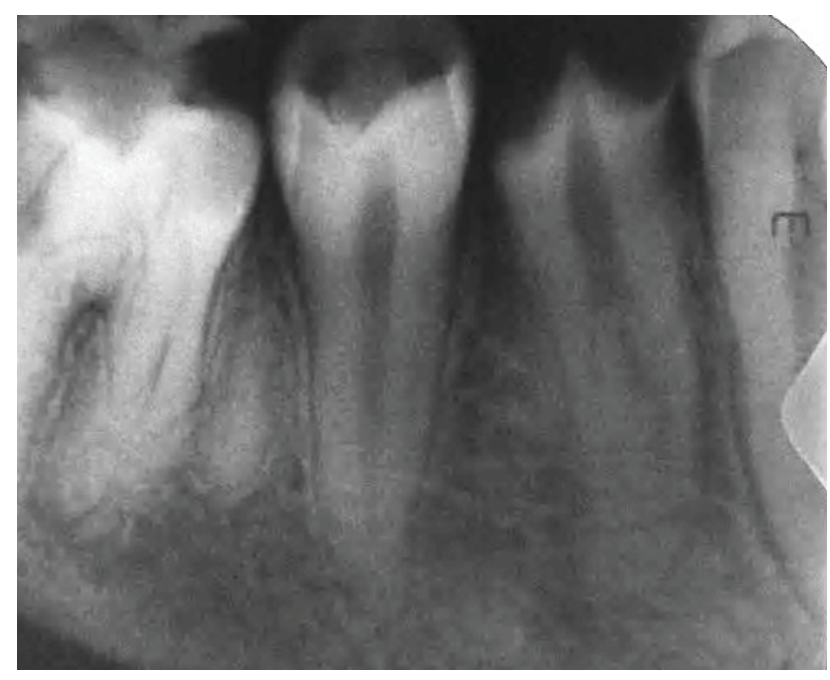

Figure 1: A preoperative periapical radiograph of tooth $\# 44$.

surface in the middle or apical third of the main canal. Such a tooth's radiographic appearance is of a visible coronal canal that seems to disappear in the middle or apical third abruptly; this phenomenon is called the fast break phenomena [15].

Slowey suggested that the mandibular premolars may present the most significant difficulty of all teeth to treat endodontically [15]. To achieve endodontic success, the entire root canal system must be debrided, disinfected, and obturated [16]. These objectives are challenging to achieve in cases with limited access and complex anatomy. Therefore, this report aimed to demonstrate the unusual anatomy of mandibular first premolar with three root canals and mandibular second premolar with four root canals and the successful endodontic management despite the limited available resources.

\section{Case 1}

A 46-year-old male Saudi patient presented to the Endodontic clinic of Albaha Dental Center in Albaha city of Saudi Arabia, with a chief complaint of pain in the region of lower right posterior teeth for one week. The pain was intermittent that intensified by cold stimuli. The patient's medical history was noncontributory.

The clinical examination revealed a carious mandibular right first premolar and first molar. Tooth \#44 and \#46 were not mobile, and periodontal probing was within physiological limits. The cold test revealed a lingering response with tooth \#44 and a normal response with tooth \#46, and both teeth were not tender on percussion. A preoperative radiograph revealed coronal radiolucency approaching the pulp of tooth \#44. Furthermore, the fast break phenomenon indicated the presence of division of the main canal into more than one canal at the middle root level (Figure 1). A diagnosis of symptomatic irreversible pulpitis with normal periapex was made, and nonsurgical root canal treatment was planned based on the clinical and radiographic findings.

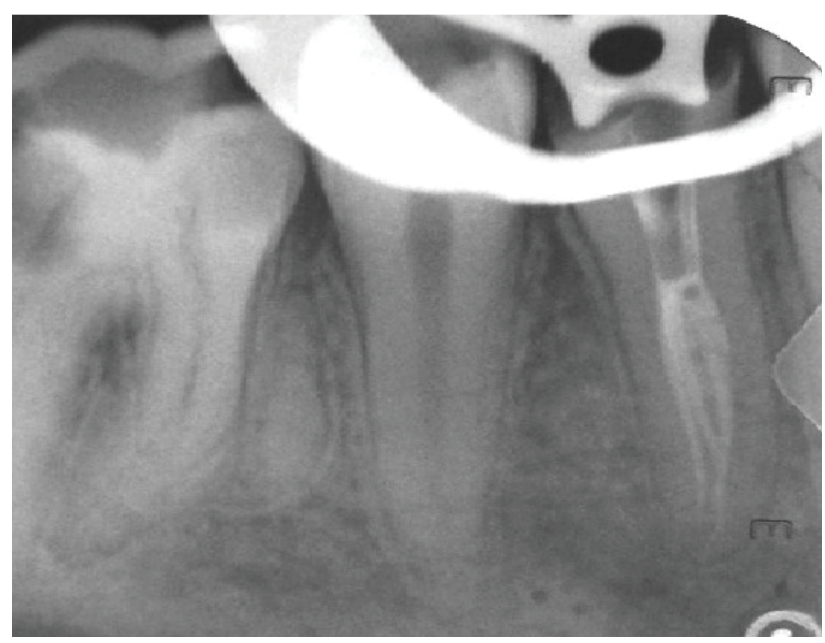

Figure 2: Mid condensation periapical radiograph of tooth \#44.

The treatment plan was discussed with the patient, and the patient signed the consent form.

After administration of local anesthesia ( $2 \%$ xylocaine with 1:100,000 adrenaline) and the rubber dam application, complete caries excavation and access were gained with No. 4 round bur (Dentsply Maillefer, Ballaigues, Switzerland). The access cavity was modified to be wider to gain sufficient access to the canals. Then, the access cavity was thoroughly irrigated with $5 \% \mathrm{NaOCl}$. Clinical examination using a DG-16 endodontic explorer (HuFriedy, Chicago, IL) with the aids of the magnifying loups $(3.5 \times 420 \mathrm{~mm})$ and LED headlight revealed three root canals: Mesiobuccal, distobuccal, and lingual. the root canals configuration was a type II according to Gulabivala and colleagues classification as shown in (Figure 2) [17].

The coronal third of the main canal was prepared using Gates Glidden, size 3 and 4 (Dentsply Maillefer, Ballaigues, Switzerland). The working length was then determined radiographically (lost radiograph) and confirmed using an apex locater (Root zx II, MORITA, JAPAN). Canals were then cleaned and shaped using Rotary endodontic file PROFILE ${ }^{\circledR}, 4 \%$ taper (Dentsply Maillefer, USA) in descending manner (step down). The master file size was \#30 Profile with $4 \%$ taper. The root canals were thoroughly irrigated with $5 \%$ sodium hypochlorite solution (20 cc) and $17 \%$ ethylenediaminetetraacetic acid $(10 \mathrm{cc})$. Root canals patency was maintained throughout the cleaning and shaping steps using size $10 \mathrm{~K}$-file (Dentsply Maillefer, Ballaigues, Switzerland). Root canal filling was performed using matching gutta-percha cones (Dentsply Maillefer, USA) and AH Plus sealer (Dentsply DeTrey GmbH, USA). The sealer was introduced into the canals with \#20 K-file, rotated in an anticlockwise direction. Then vertical compaction was performed up to the canal's trifurcation (Figure 2 and Figure 3).

\section{Case 2}

A 29-year-old male Saudipatient was referred to the 


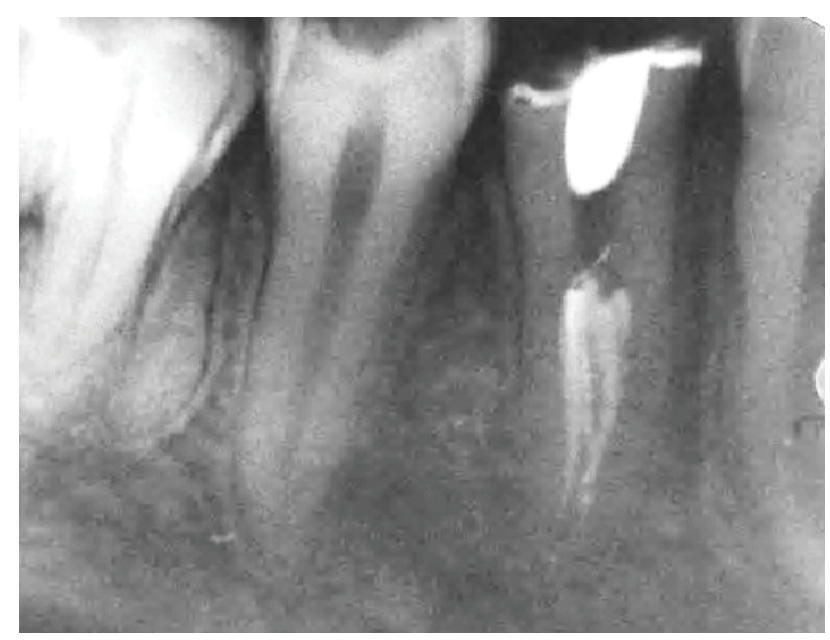

Figure 3: Obturation radiograph of tooth \#44.

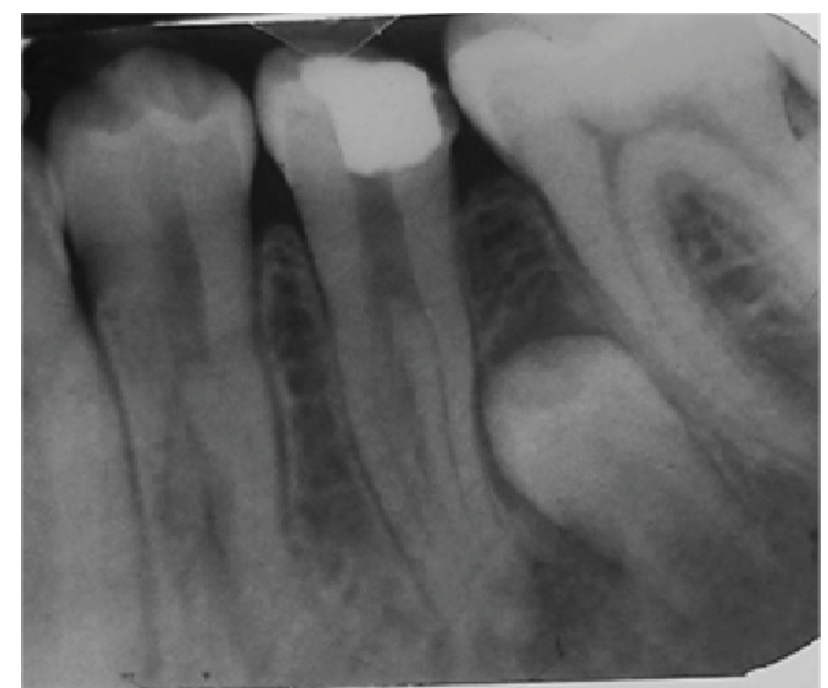

Figure 4: A preoperative periapical radiograph of tooth \#35.

Endodontic clinic of Albaha Dental Center to complete the root canal treatment of tooth \#35 that was initiated previously in the primary health care clinic. The offending tooth was asymptomatic. The patient's medical history was noncontributory.

A careful clinical examination revealed a temporary filling, including occlusal and distal surfaces of tooth \#35. Tooth \#35 was not mobile, and periodontal probing was within physiological limits. Electric pulp testing and cold test were inapplicable, and the tooth was feeling different on percussion. A preoperative radiograph showed radiopaque filling material in the pulp space. It also showed apical radiolucency related to both roots of tooth \#35 (Figure 4). Impacted supernumerary premolar was noticed the area between the mesial root of tooth \#36 and the distal root of tooth \#35. A pulpal diagnosis of previously initiated therapy with asymptomatic apical periodontitis was made. Therefore, a nonsurgical root canal treatment was planned. The treatment plan was discussed with the patient, and the patient signed the consent form.

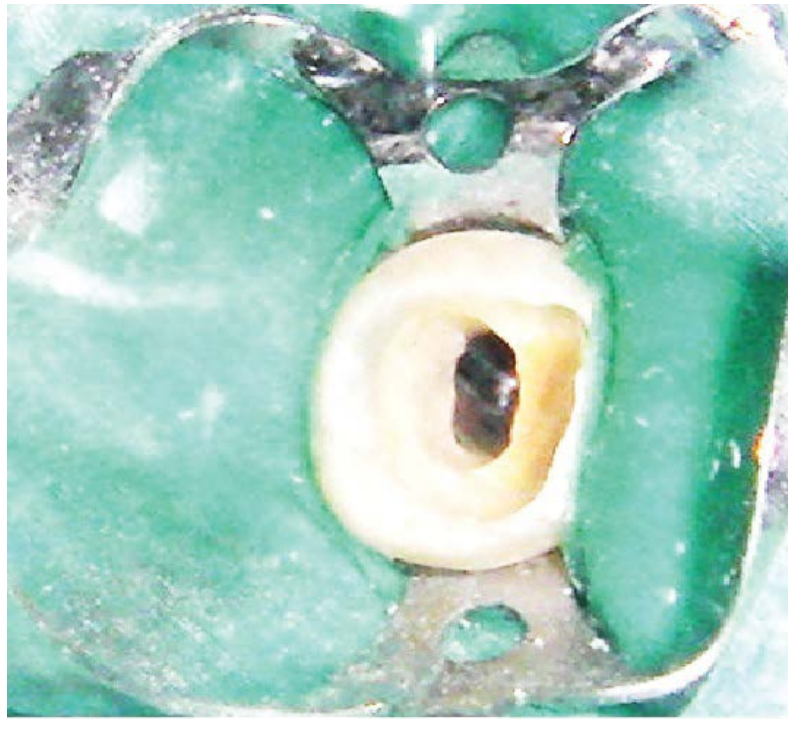

Figure 5: Access cavity of tooth \#35.

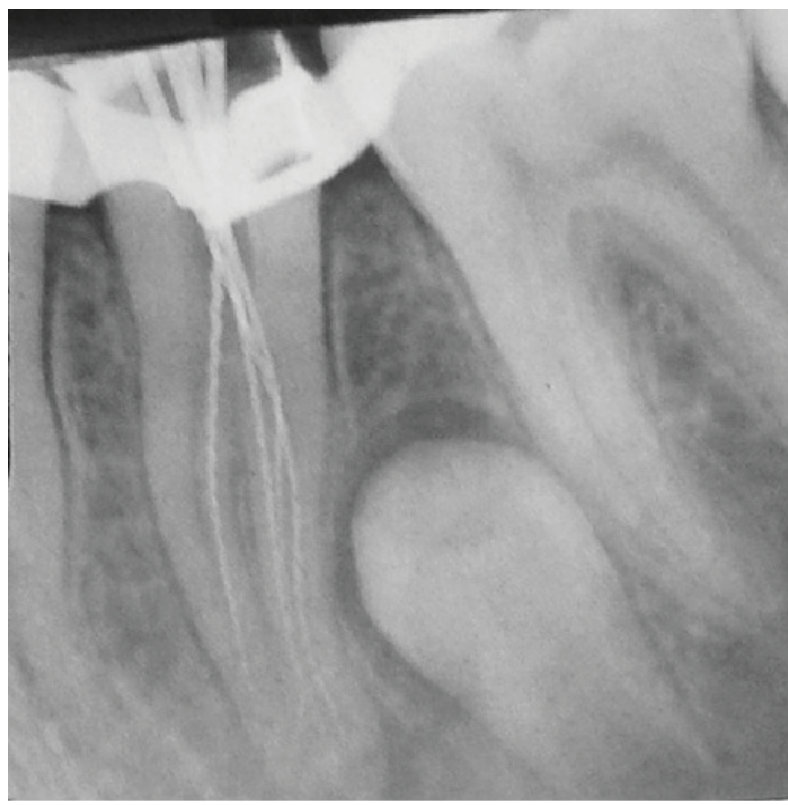

Figure 6: Working length radiograph of tooth \#35.

After local anesthesia administration (2\% xylocaine with 1:100,000 adrenaline), the access was regained with No. 4 round bur (Dentsply Maillefer, Ballaigues, Switzerland) under rubber dam isolation. The access opening using Gates Glidden size 3 and 4 (Dentsply Maillefer, Ballaigues, Switzerland) to gain better access to the orifices of the canals. The access cavity was then thoroughly rinsed with $5 \% \mathrm{NaOCl}$. Clinical examination using a DG-16 endodontic explorer (Hu-Friedy, Chicago, IL) with the aids of the magnifying loupes $(3.5 \times 420$ $\mathrm{mm}$ ) and LED headlight. Four orifices were identified: Mesiobuccal, distobuccal, lingual, and middle orifice. The middle orifice appearance mimics the root canal orifices usually seen in the C-shaped root canals (Figure 5).

The working length was determined using an apex locater (Root ZX II, MORITA, JAPAN) and confirmed radiographically (Figure 6). Root canals were then prepared 


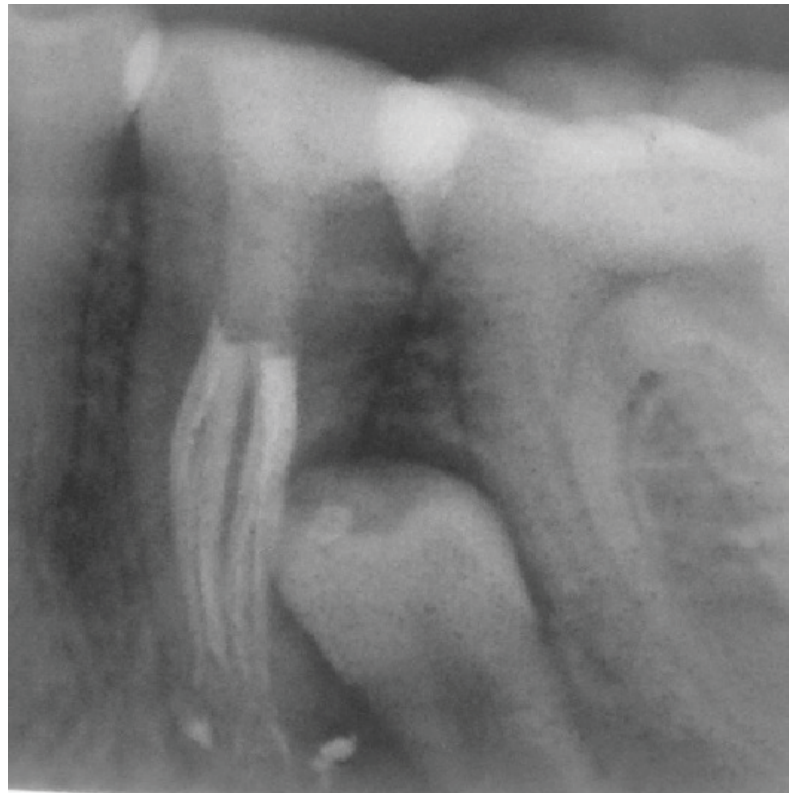

Figure 7: Distal angle periapical radiograph of tooth \#35 after obturation.

with Rotary endodontic file PROFILE ${ }^{\circledR}, 4 \%$ taper (Dentsply Maillefer, USA) using the step-down technique. The master file size was \#25 Profile with 4\% taper. The canals were thoroughly irrigated with $5 \% \mathrm{NaOCl}$ solution (20 cc) and 17\% ethylenediaminetetraacetic acid (10 cc). Root canals patency was maintained throughout the cleaning and shaping steps using size $10 \mathrm{~K}$-file (DentspIy Maillefer, Ballaigues, Switzerland). Root canal filling was done using matching gutta-percha cones (DentspIy Maillefer, USA) and AH Plus sealer (Dentsply DeTrey $\mathrm{GmbH}, \mathrm{USA}$ ). The sealer was introduced into the canals by \#20 K-file rotated in an anticlockwise direction. Then vertical compaction was performed to the level of the canal's trifurcation (Figure 7 and Figure 8). The root canal anatomy configuration is class VI according to Gulabivala and colleagues classification [17]. Root canal sealer puff was noticed apically and laterally in conjunction with the impacted premolar.

\section{Discussion}

Complexities of the mandibular premolars root canal anatomy have been shown in the literature to be not uncommon findings [1,2]. However, few case reports of the mandibular premolars had been published showing three root canals, and fewer reports had been published for lower premolars with four root canals [18]. Root canal anatomy aberrations of the mandibular premolars, including the number of canals, canal configurations, taurodontism, c shaped root, and apical delta, might compromise the root canal treatment procedures.

Based on the two-dimensional radiographic images of the two cases reported, the radiographic findings suggested the presence of $\mathrm{C}$-shaped mandibular premolars. Cone-beam computed tomography (CBCT) is a useful image to confirm a $\mathrm{C}$-shaped root canal system

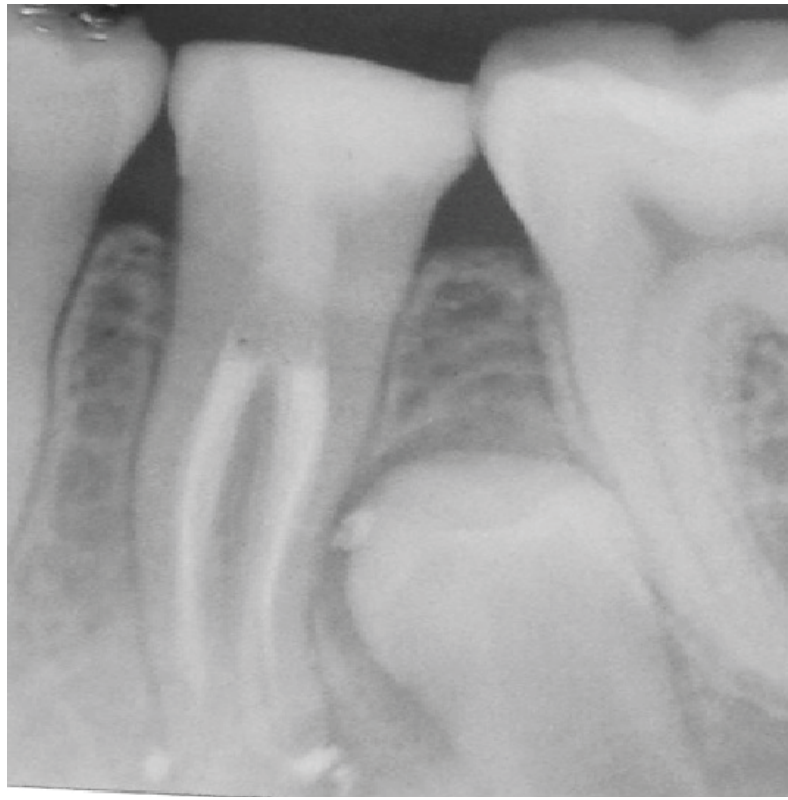

Figure 8: Head on periapical radiograph of tooth \#35 after obturation.

$[19,20]$. CBCT images provide three-dimensional imaging that enables the operator to study the anatomical structures and the pathological changes with more precise details. However, in this case, CBCT images were not taken because it was not available in the Albaha region at the time of treatment. Furthermore, the canals orifices orientations indicate $\mathrm{C}$-shaped canal, as shown in Figure 5.

A sudden narrowing of the root canal space known as "the fast break phenomena" indicates its division into two or more canals $[2,15]$. This was evident in both cases preoperative radiographs. Pulp chamber map analysis with the aids of the magnifying loupes helps in locating all the root canals.

The use of dental magnifying loops enhances a better visualization to determine the extra root canals [21]. Moreover, fiber optic illumination and the bubbling effects with sodium hypochlorite would help explore the complex toot canal system [22].

Modification of the access cavity using Gates Glidden to enhance the root canals' visualization and accessibility is recommended for cases with weird root canal anatomy. Anatomical studies of the mandibular premolars with three canals have shown one orifice in the lingual side and two in the buccal [1]. The first case in this report was in accordance with their findings. Electronic apex locators have been used to determine and confirm the working length. It was proven to be an accurate tool in working length determination, especially in complex anatomy cases [23].

It is a fact that increasing the apical preparation size leads to more debridement of root canals and better disinfection of the critical apical third [24]. However, in the complex root canal anatomy such as these two cas- 
es, it is advocated to keep the apical third's mechanical preparation as small as possible to decrease the chances of iatrogenic preparation errors.

The use of the standardized gutta-percha cones and lateral compaction might not be applicable in cases with limited access and complex anatomy. Therefore, all the root canals were obturated using matching gutta-percha cone and vertical compaction technique followed by coronal restoration in order to decrease the chances of leakage [25].

Enough anatomical knowledge background, detailed clinical investigations, proper utilization of the radiographic imaging, magnification tools, and additional lighting are necessary for best visualization, understanding, and management of the mandibular premolars. After collection and analysis of all the clinical and radiographic data, it is always recommended to assess the level of difficulty of the cases and then to treat or refer to the specialist if needed.

\section{Conclusion}

The mandibular premolars anatomy has complex variations. Enough anatomical knowledge background, detailed clinical investigations, proper utilization of radiographic imaging, and magnification tools are mandatory for best understanding, visualization, and managing the mandibular premolars.

\section{References}

1. Kottoor J, Albuquerque D, Natanasabapathy V, Kuruvilla J (2013) Root anatomy and root canal configuration of human permanent mandibular premolars: A systematic review. Anat Res Int 2013: 254250.

2. Vertucci FJ (1978) Root canal morphology of mandibular premolars. J Am Dent Assoc 97: 47-50.

3. Ordinola-Zapata R, Monteiro Bramante C, Gagliardi Minotti P, Cavalini Cavenago B, Gutmann JL, et al. (2015) Micro-CT evaluation of c-shaped mandibular first premolars in a Brazilian subpopulation. Int Endod J 48: 807-813.

4. Geider P, Perrin C, Fontaine M (1989) Endodontic anatomy of lower premolars-apropos of 669 cases. J Odontol Conserv 10: 11-15.

5. Iyer VH, Indira R, Ramachandran S, Srinivasan MR (2006) Anatomical variations of mandibular premolars in Chennai population. Indian J Dent Res 17: 7-10.

6. Sert S, Bayirli GS (2004) Evaluation of the root canal configurations of the mandibular and maxillary permanent teeth by gender in the Turkish population. J Endod 30: 391-398.

7. Shalavi S, Mohammadi Z, Abdolrazzaghi M (2012) Root canal treatment of maxillary and mandibular three-rooted premolars: Case reports. Iran Endod J 7: 161-164.

8. Chan K, Yew SC, Chao SY (1992) Mandibular premolar with three root canals-two case reports. Int Endod J 25: 261-264.

9. Gu Y, Zhang Y, Liao Z (2013) Root and canal morphology of mandibular first premolars with radicular grooves. Arch Oral Biol 58: 1609-1617.

10. Fan B, Ye W, Xie E, Wu H, Gutmann JL (2012) Three-di- mensional morphological analysis of c-shaped canals in mandibular first premolars in a Chinese population. Int Endod J 45: 1035-1041.

11. Chen J, Li X, Su Y, Zhang D, Wen X, et al. (2015) A micro-computed tomography study of the relationship between radicular grooves and root canal morphology in mandibular first premolars. Clin Oral Investig 19: 329-334.

12. Trope M, Elfenbein L, Tronstad L (1986) Mandibular premolars with more than one root canal in different race groups. J Endod 12: 343-345.

13. Vertucci FJ, Haddix JE (2011) Tooth morphology and access cavity preparation. In: Hargreaves KM, Cohen S, Berman LH, Cohen's pathways of the pulp. (10 $10^{\text {th }}$ edn $)$, Mosby Elsevier, 136-222.

14. Woelfel J, Scheid R (2002) Dental anatomy its relevance to dentistry. Philadelphia: Lippincott williams \& wilkins.

15. Slowey RR (1974) Radiographic aids in the detection of extra root canals. Oral Surg Oral Med Oral Pathol 37: 762772.

16. Weine FS (1995) Nonsurgical re-treatment of endodontic failures. Compend Contin Educ Dent 16: 326-335.

17. Gulabivala K, Aung TH, Alavi A, Ng YL (2001) Root and canal morphology of burmese mandibular molars. Int Endod J 34: 359-370.

18. Al-Fouzan KS (2001) The microscopic diagnosis and treatment of a mandibular second premolar with four canals. Int Endod J 34: 406-410.

19. Yoshioka T, Villegas JC, Kobayashi C, Suda H (2004) Radiographic evaluation of root canal multiplicity in mandibular first premolars. J Endod 30: 73-74.

20. Michetti J, Maret D, Mallet JP, Diemer F (2010) Validation of cone beam computed tomography as a tool to explore root canal anatomy. J Endod 36: 1187-1190.

21. De Carlo Bello M, Tibúrcio Machado C, Dotto Londero C, Branco Barletta F, Cunha Moreira CH, et al. (2018) Diagnostic efficacy of four methods for locating the second mesiobuccal canal in maxillary molars. Iran Endod J 13: 204-208.

22. Albuquerque D, Kottoor J, Hammo M (2014) Endodontic and clinical considerations in the management of variable anatomy in mandibular premolars: A literature review. Biomed Res Int.

23. Singh SV, Nikhil V, Singh AV, Yadav S (2012) An in vivo comparative evaluation to determine the accuracy of working length between radiographic and electronic apex locators. Indian J Dent Res 23: 359-362.

24. Ørstavik D, Kerekes K, Molven O (1991) Effects of extensive apical reaming and calcium hydroxide dressing on bacterial infection during treatment of apical periodontitis: A pilot study. Int Endod J 24: 1-7.

25. Buchanan LS (1996) The continuous wave of obturation technique: 'Centered' condensation of warm gutta-percha in 12 seconds. Dent Today 15: 60-67. 